\title{
COMMENTARY
}

\section{Elongation of the epiphyseal trabecular bone in transgenic mice carrying a klotho gene locus mutation that leads to a syndrome resembling aging}

\author{
T Yamashita ${ }^{1,4}$, A Nifuji ${ }^{1}$, K Furuya ${ }^{1}$, Y Nabeshima ${ }^{2,3,4}$ and \\ M Noda ${ }^{1}$ \\ ${ }^{1}$ Department of Molecular Pharmacology, Medical Research Institute, Tokyo Medical and Dental University, Tokyo, Japan, ${ }^{2}$ Division of Molecular Genetics, \\ National Institute of Neuroscience, NCNP, Tokyo, Japan, ${ }^{3}$ Institute for Molecular and Cellular Biology, Osaka University, Osaka, Japan and ${ }^{4}$ Core Research \\ for Evolutional Science and Technology, JRDC, Tokyo, Japan \\ (Requests for offprints should be addressed to M Noda, 3-10, Kanda-Surugadai 2-Chome, Chiyoda-ku, Tokyo, 101-0062, Japan)
}

\begin{abstract}
Bone diseases such as osteoporosis and osteoarthritis are regarded as age-associated diseases, and occur in a significantly increasing number of patients, but the underlying mechanisms of these age-associated bone diseases are not yet clear. We have established a transgenic mouse line by an insertion mutation. These mice exhibit many features related to precocious aging. Homozygote mutant mice, which lack expression of the newly identified targeted gene, klotho ( $k l)$, exhibit atherosclerosis, emphysema, hypogonadism and calcification of soft tissues, and die within 3-4 months. We describe here the radiological and histological characteristics of the skeletal abnormalities in the bones of the mice with a mutation in the $k l$ gene locus. In heterozygous mice $(+/ k l)$, the skeletal patterns and structures remain normal and most features are similar to those in the wild-type, whereas histological examinations of homozygous mice $(\mathrm{kl} / \mathrm{kl})$ show abnormal elongation of the trabecular bone(s) in the epiphyses of long bones. As with their long bones, on radiographic examination the
\end{abstract}

mid parts of the vertebral bones of these mice show less radiopacity compared with the wild-type, again resembling human vertebrae of osteoporotic patients. The elongation of the trabecular bones results in high radiopacity on both ends of each of the vertebrae, and in the epiphyses of the long bones. Cancellous bone volume in the epiphyses of the homozygote mice is three times that of the wild-type mice. The $k l / k l$ mice are smaller than the wild-type litter mates and hence the size of their long bones is less than that of the wild-type litter mates. These observations, and the osteopenia in the vertebrae and long bones in these mice, suggest the presence of abnormality in bone metabolism, the elongation of the trabecular bone apparently resulting from the relatively low levels of bone resorption. Therefore, the $\mathrm{kl} / \mathrm{kl}$ mutant mice could serve as an interesting tool to study the effects of the lack of the product of the new gene, klotho, on bone metabolism.

Journal of Endocrinology (1998) 159, 1-8

\section{Introduction}

Aging is a complex phenomenon that remains of major scientific interest. However, the molecular mechanisms of aging that limit life-span are not well understood. Many environmental and epigenetic factors could exert an influence, and some of them would promote aging. For instance, several studies using mouse models reported the involvement of immune function, which is the first line of defense against environmental insults in animals (Salazar et al. 1995). It has also been hypothesized that the high environmental concentration of oxidants promotes aging and could become a major causative factor for senescence (Ames et al. 1993).

Genetic analyses propose several molecular causes of aging, including telomeric shortening, loss of silencing, accumulation of DNA damage and defects in ribosomal DNA transcription or maintenance (Guarente 1996). These causes are related to structural alterations in the chromosomes, but the nature of the molecular events having crucial roles in aging, and which particular genes are influenced by such chromosomal alteration, have not yet been determined. Aging in individuals correlates closely with cellular senescence, but molecular 
mechanisms connecting directly individual aging and cellular senescence have not been identified.

In humans, many diseases associate with aging; however, the identities of the molecules that link these diseases and aging are not yet fully known. Precocious aging has been observed in ataxia telangiectasia, Werner's syndrome and Cockayne syndrome, and some of the genes causing these diseases have been cloned and identified (Troelstra et al. 1992, Henning et al. 1995, Savitsky et al. 1995, Barlow et al. 1996, Yu et al. 1996). These molecules are modifiers of DNA structures and, again, the final target genes of these modifiers are not yet clear.

Major bone diseases such as osteoporosis and degenerative osteoarthritis and osteomalacia are associated with aging. Although not directly linked to aging, aging-related hypercalcification shares some features with agingassociated bone diseases with regard to the unphysiological formation of ectopic calcified mass. One model of such hypercalcification is the matrix $\gamma$-carboxyglutamic acidcontaining (GLA) protein (Mgp)-deficient mouse. These mice were reported to exhibit pathological calcification of cartilages and arteries (Luo et al. 1997). Mgp is a member of mineral-binding GLA proteins and the phenotypes of the null mutant mice suggest that Mgp is an inhibitor of calcification of soft tissues. Although arterial and cartilaginous calcification in these Mgp-null mice resembles hypercalcification in aged patients, its relationship to aging has not yet been determined.

We have recently established a transgenic mouse line by an insertion mutation (Kuro-o et al. 1997). The mice exhibit many features of precocious aging and ectopic calcification. Homozygous mutant mice, which lack expression of the newly identified targeted gene, klotho $(k l)$, exhibit rapid onset of aging-related phenotypes, including atherosclerosis, emphysema, hypogonadism, calcification of soft tissues and diaphyseal osteopenia, and die within 3-4 months. The klotho gene encodes a novel membrane-bound molecule with two repetitive, enzymelike sequences, and its isoform may be released into the circulation, as shown by the presence of an alternatively spliced mRNA that lacks a transmembrane domain (Kuro-o et al. 1997). This review describes the effects of $\mathrm{kl} / \mathrm{kl}$ mutation on the trabecular bones, which are subject to dynamic remodeling in rodent bones.

\section{High radiopacity in the metaphyseal regions of $k l / k l$ mutant mice}

We previously found that the body weight of wild-type mice increased continuously during a 4 -week period of examination, whereas the body weight of $k l / k l$ mice increased in a fashion similar to that in the wild-type up to 2 weeks post partum, but did not increase as quickly as the wild-type thereafter (Fig. 1) (Kuro-o et al. 1997). Radiographs of the homozygous mice $(k l / k l)$ revealed an increase

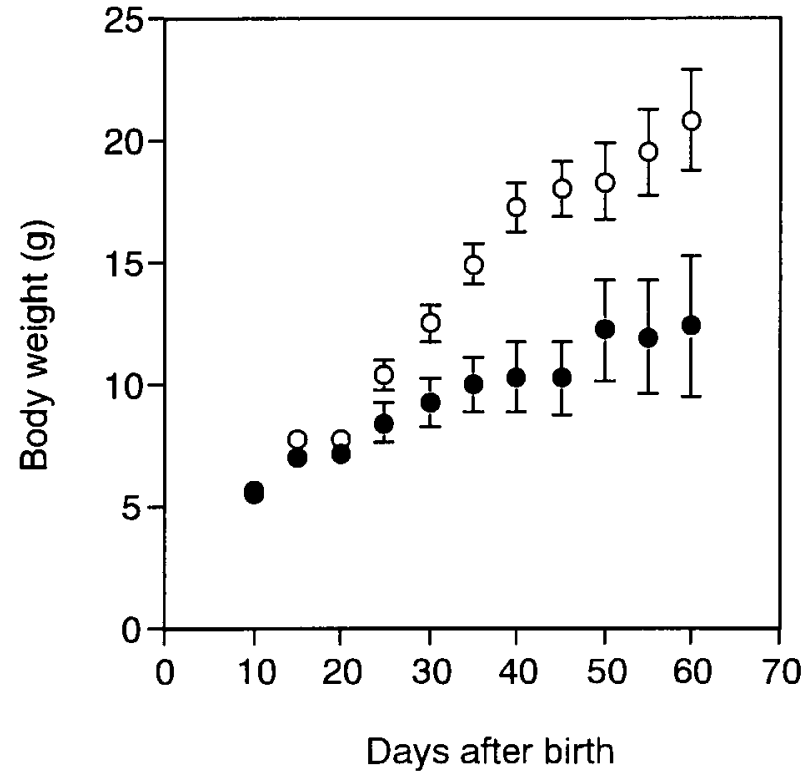

Figure 1 Time-course of changes in body weight of control and $\mathrm{kl} / \mathrm{kl}$ mice. Each point represents the averaged value of seven control mice $(\bigcirc)$ or five $\mathrm{kl} / \mathrm{kl}$ mice $(\bullet)$. Bars represent S.E.M.

in bone density on both rostal and caudal ends of the vertebrae (Fig. 2A, black arrow heads), associated with low radiopacity in the mid-regions (Fig. 2A, white arrow head) (Yamashita et al. 1998). High radiopacity was also seen on both ends of the long bones (Fig. 2C, arrow heads). The mutant mice also showed slight but consistent irregularity in the contour of the ribs (Fig. 2E) compared with the corresponding regions of the wild-type animals (Fig. 2F).

Radiographic examination indicated that increased radiopacity in the metaphyses of the bones in the $\mathrm{kl} / \mathrm{kl}$ homozygote mutant mice was due to aberrant elongation of the trabeculae in the bones, including vertebrae, femoral, humeral, metacarpal and metatarsal bones (Fig. 2C, D). These bones contain cancellous bones at their metaphyseal region. In contrast, morphology of the calvariae, which consist mainly of cortical bones, was similar between $k l / k l$ mutant and wild-type mice (Fig. 2G and $\mathrm{H}$ respectively).

\section{Elongation of trabecular bones in $k l / k l$ mutant mice}

Histological examination also showed the elongation of the trabeculae in these $k l / k l$ homozygous mutant mice (Fig. 3A, C) compared with the controls (Fig. 3B, D). The area stained deep blue by toluidine blue-O was more extensive in the trabeculae of $\mathrm{kl} / \mathrm{kl}$ mutant mice (Fig. 3A) than in those of wild-type animals (Fig. 3B).

Histomorphometry based on computerized multicolorimage analysis (Nireco system) in the metaphyseal regions 

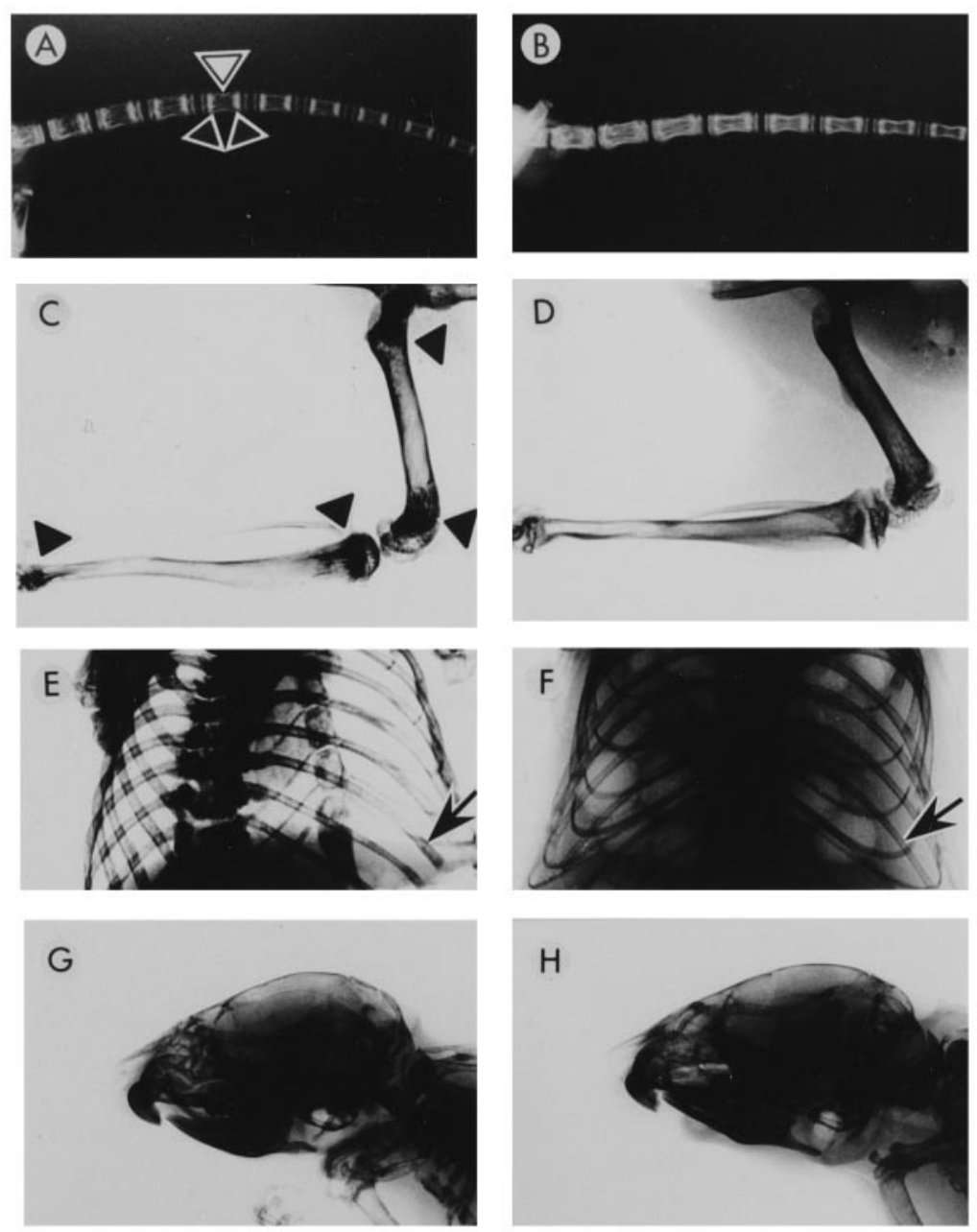

Figure 2 Radiographs of the tail vertebrae (A and $B)$, femora and tibiae (C and $D)$, ribs ( $\mathrm{E}$ and $\mathrm{F}$ ), calvariae $(\mathrm{G}$ and $\mathrm{H}$ ) of $\mathrm{kl} / \mathrm{kl}$ mutant mice $(\mathrm{A}, \mathrm{C}, \mathrm{E}, \mathrm{G})$ and wild-type mice $(B, D, F, H)$. Bone density is increased in the radiographs of the end-regions of the bones in $k l / k l$ mice (A, C). $k l / k l$ mutants show hypercalcification on both caudal and rostal ends of all the vertebrae (A). Primary trabeculae in the metaphyses of the long bones also show aberrant elongation of calcified trabeculae (C). Several transgenic mice show thickening and irregular contour in bony ribs (E). Calvariae are similar between mutant and control mice ( $\mathrm{G}$ and $\mathrm{H}$ ). Black arrow heads, increased radiodensity in the metaphyses of the bones; open arrow head, reduced radiopacity. The arrows in (E) and (F) point to the contour of the ribs

of tibiae, using undecalcified histological sections stained with toluidine blue, indicated that total bone volume was three- to fourfold greater in the mutant mice compared with the wild type (46.3\% and $12 \cdot 7 \%$ respectively; $P<0 \cdot 01$ (Fig. 3A, B; Table 1)). The area of the deep-blue-stained matrices (most probably corresponding to the regions of the calcified cartilaginous trabeculae) in the trabeculae of the $\mathrm{kl} / \mathrm{kl}$ mutant mice was also three- to fourfold more than that of controls. Accordingly, the ratios between the areas stained deep blue and the total trabecular bone volume were similar (48.3\% and $46 \cdot 9 \%$ respectively, in the $k l / k l$ mutant and the control mice; Table 1), indicating that the levels of calcification of at least the primary trabeculae are normal in $\mathrm{kl} / \mathrm{kl}$ mutant mice.

To examine the osteoid area, we stained undecalcified sections of the tibiae according to the Villanueva method. Histomorphometry again revealed elongation of the trabeculae in $k l / k l$ mutant mice (Fig. 3C, D). The total trabecular bone volume/tissue volume ratio was increased about threefold in $\mathrm{kl} / \mathrm{kl}$ mutant mice compared with the wild-type $(35 \cdot 5 \%$ compared with $12 \cdot 7 \%, P<0 \cdot 01$; Table 1). The osteoid area, which stained red, was larger 

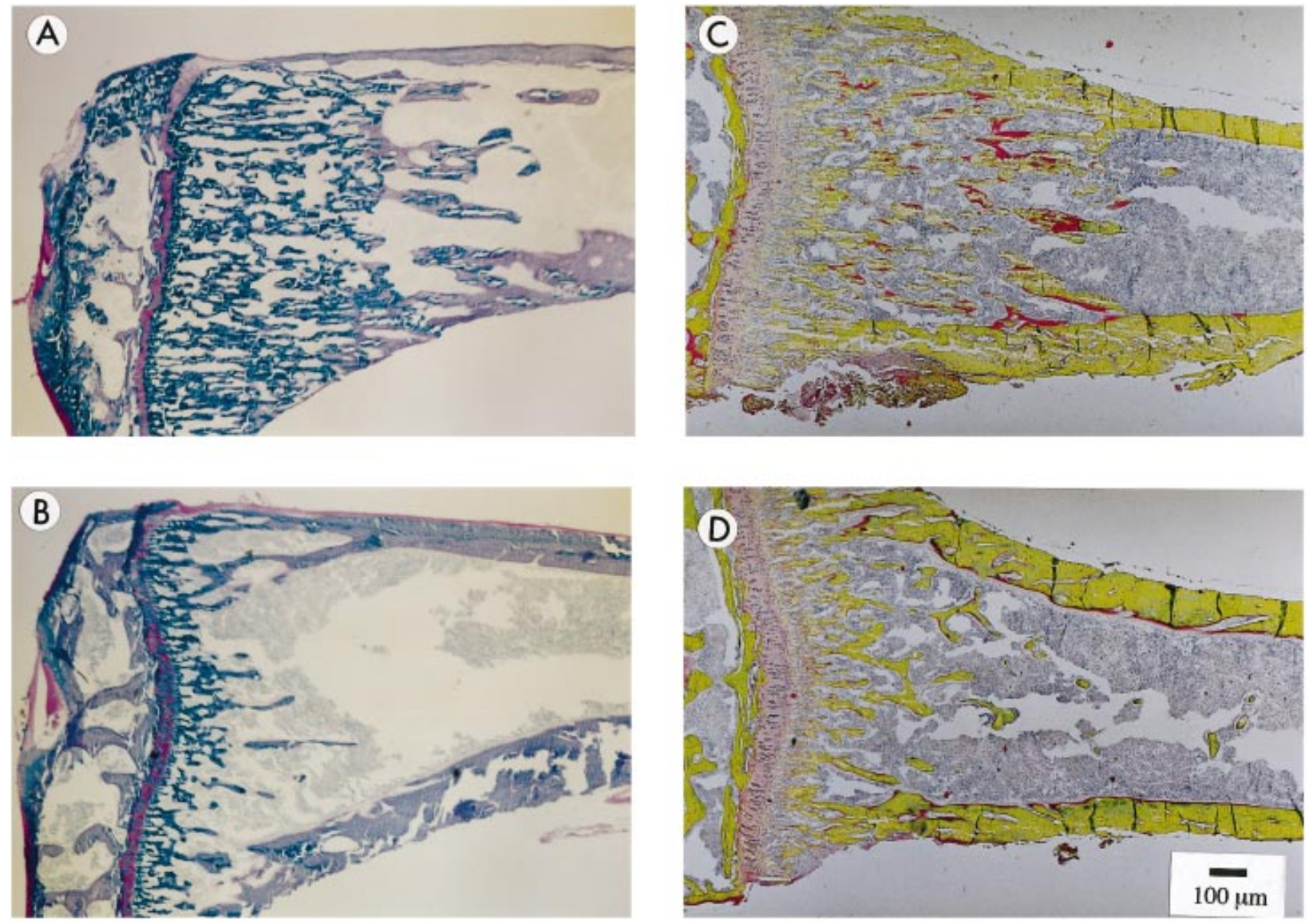

Figure 3 Histology of the tibiae of $k l / k l$ mutant mice. Toluidine blue staining of the sections for tibiae of 4 -week old $k l / k l$ (A) and wild-type (B) mice, and Villanueva staining of the tibiae of $k l / k l$ (C) and wild-type (D) mice. Bar represents $100 \mu \mathrm{m}$.

in $\mathrm{kl} / \mathrm{kl}$ mutant mice (Fig. 2C) than in the wild-type animals (Fig. 2D), and quantitation also indicated this increase in osteoid thickness in $\mathrm{kl} / \mathrm{kl}$ mutant mice compared with the wild-type $(18.2 \mu \mathrm{m}$ compared with $7 \cdot 9 \mu \mathrm{m}, P<0 \cdot 01$; Table 1), suggesting either that bone formation per se is slightly accelerated in klotho mutant mice or that calcification of the bones in the secondary trabecular may be slightly retarded.

Skeletal abnormality in $\mathrm{kl} / \mathrm{kl}$ mice was observed in the trabeculae of the long bones and vertebrae, but not in calvariae. The reason for this is not clear. The difference may reflect the different pathways of formation of these bones. Calvariae are formed via membranous bone formation and consist mainly of cortical bones, whereas long bones and vertebrae are formed via endochondral bone formation and contain more cancellous bones. Cancellous bones are known to be more active in terms of their turnover than are the cortical bones in calvaria, thus possibly making the cancellous bones more susceptible to the changes induced by this mutation.
Table 1 Results of bone morphometry (undecalcified section). Values are means \pm S.E.M.

\section{Genotype}

\begin{tabular}{|c|c|}
\hline$k l / k l(n=6)$ & Wild-type $(n=4)$ \\
\hline $46 \cdot 3 \pm 4 \cdot 2^{\star}$ & $12 \cdot 7 \pm 0 \cdot 6$ \\
\hline $22 \cdot 3 \pm 2 \cdot 1^{*}$ & $6 \cdot 0 \pm 0 \cdot 5$ \\
\hline $48 \cdot 3 \pm 1 \cdot 6$ & $46 \cdot 9 \pm 4 \cdot 5$ \\
\hline $35 \cdot 5 \pm 4 \cdot 4^{*}$ & $12 \cdot 7 \pm 0 \cdot 6$ \\
\hline $4 \cdot 5 \pm 1 \cdot 8$ & $3 \cdot 5 \pm 0 \cdot 7$ \\
\hline $18 \cdot 2 \pm 3 \cdot 0^{*}$ & $7 \cdot 9 \pm 0 \cdot 1$ \\
\hline $39 \cdot 4 \pm 3 \cdot 9^{*}$ & $27 \cdot 8 \pm 1 \cdot 9$ \\
\hline
\end{tabular}

Morphometric parameter

Toluidine blue staining

$\mathrm{BV} / \mathrm{TV}(\%)$

DT/TV $(\%)$

DT/BV (\%)

Villanueva staining

$\begin{array}{lcr}\text { BV/TV }(\%) & 35 \cdot 5 \pm 4 \cdot 4^{*} & 12 \cdot 7 \pm 0 \cdot 6 \\ \text { OV/TV }(\%) & 4 \cdot 5 \pm 1 \cdot 8 & 3 \cdot 5 \pm 0 \cdot 7 \\ \text { O.Th }(\mu \mathrm{m}) & 18 \cdot 2 \pm 3 \cdot 0^{*} & 7 \cdot 9 \pm 0 \cdot 1 \\ \text { Tb.Th }(\mu \mathrm{m}) & 39 \cdot 4 \pm 3 \cdot 9^{*} & 27 \cdot 8 \pm 1 \cdot 9\end{array}$

$\mathrm{BV} / \mathrm{TV}$, total trabecular bone volume per tissue volume; DT/TV, deep-blue-stained bone volume per tissue volume; DT/BV,

ratio of deep-blue-stained bone volume to total bone trabeculae; OV/TV, osteoid volume per tissue volume; O.Th, thickness of osteoid; Tb.Th, thickness of trabeculae. There is a considerable increase in trabecular bone volume of tibiae in $\mathrm{kl} / \mathrm{kl}$ homozygotes compared with that in wild-type mice. ${ }^{*}$ Significant difference $(P<0 \cdot 01)$ compared with wild-type. 

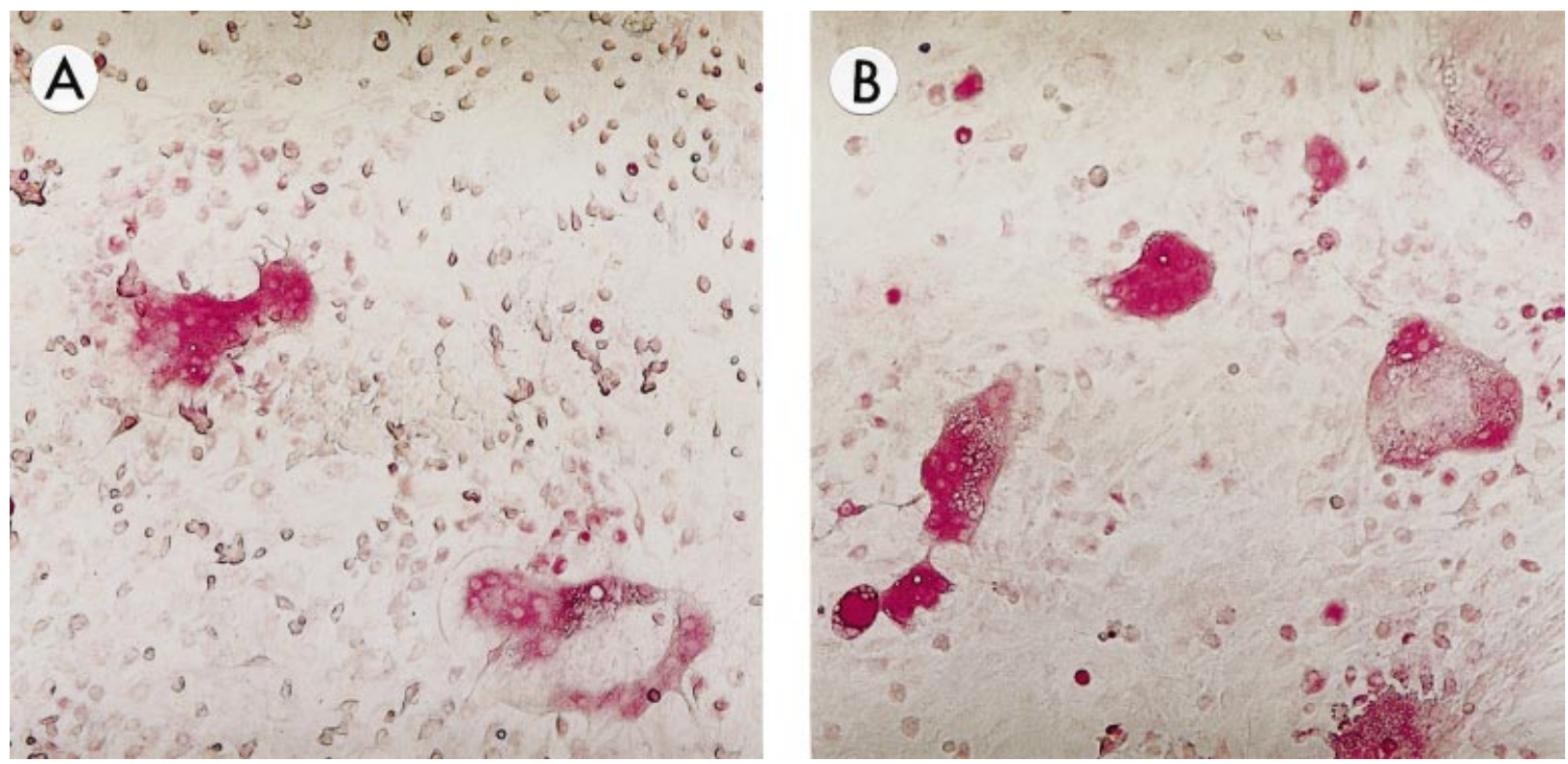

Figure 4 Development of TRAP-positive MNCs in the cocultures of cells prepared from $k l / k l$ mutant or wild-type mice. Cocultures were conducted in the presence of 1,25-dihydroxyvitamin $D_{3}$ for 14 days using 96 -well plates $\left(0.32 \mathrm{~cm}^{2} /\right.$ well). The cells were stained for TRAP; positive cells appear red. (A) Primary cultures of osteoblasts $\left(2 \times 10^{3}\right.$ cells/well) isolated from $\mathrm{kl} / \mathrm{kl}$ mutant mice calvaria were cocultured with bone marrow cells $\left(1 \times 10^{4}\right.$ cells/well) isolated from $\mathrm{kl} / \mathrm{kl}$ mutant mice. (B) Primary cultures of osteoblasts $\left(2 \times 10^{3}\right.$ cells/well) isolated from wild-type mice calvaria were cocultured with bone marrow cells $\left(1 \times 10^{4}\right.$ cells/well) isolated from wild-type mice. The morphology and number of TRAP-positive MNCs are similar between the cocultures using cells from either $\mathrm{kl} / \mathrm{kl}$ mutant and wild-type mice.
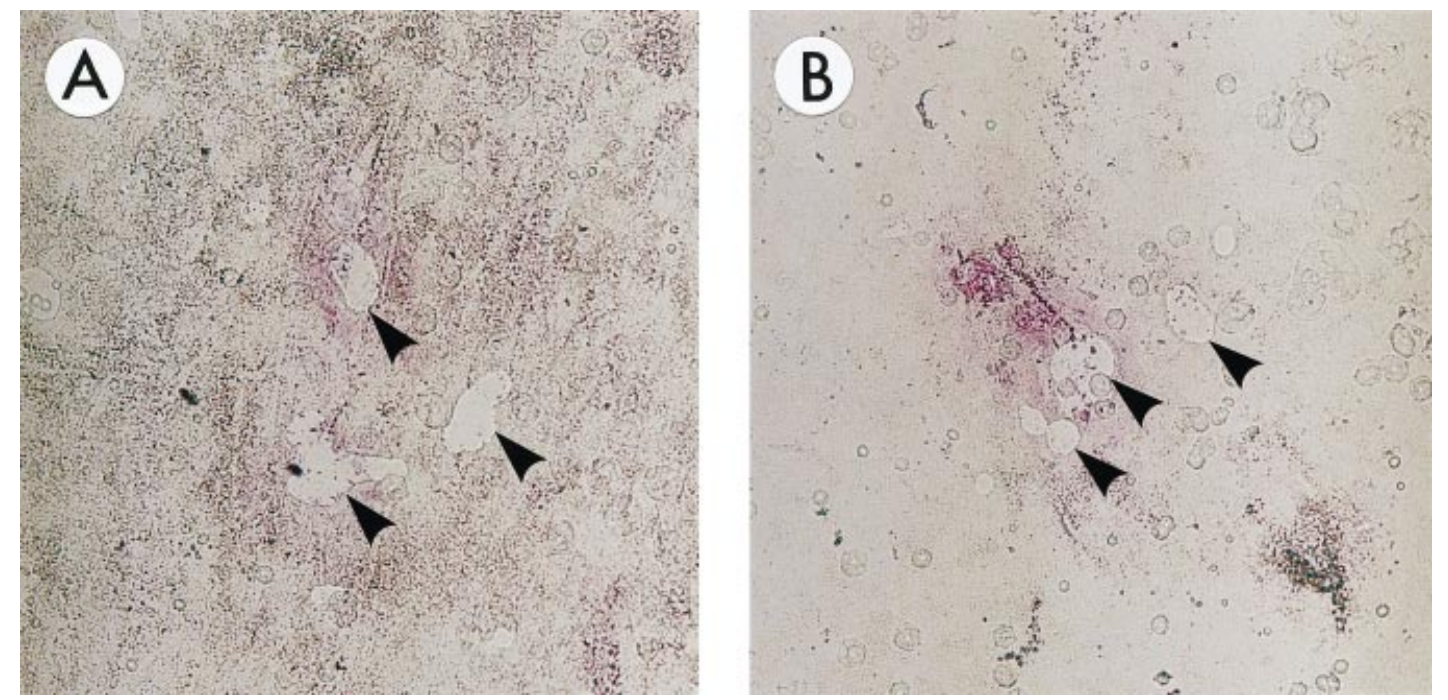

Figure 5 Pit assay using TRAP-positive osteoclast-like cells from (A) $k l / k l$ mutant mice and (B) wild-type mice. To estimate resorbing activity of the TRAP-positive osteoclast-like cells developed in vitro as shown in Fig. 4, cocultures were conducted as described in the legend to Fig. 4, except that the cells were cultured on hydroxyapatite-coated wells $\left(0.32 \mathrm{~cm}^{2} /\right.$ well) for 1 week. The cells were removed from the surface for examination. Open areas (arrow heads) indicate the resorption of hydroxyapatite by the osteoclast-like cells.

Quantitative analyses of the histological sections indicated about three-fold more trabecular bone volume in the proximal metaphyses of the tibiae in $\mathrm{kl} / \mathrm{kl}$ mutants than in wild-type mice (Table 1). These histomorphological characteristics suggest that certain steps in the pathway to maintain the volume of the trabecular bone 
could be critical targets of the effects of the mutation in these transgenic mice. Increase in the trabecular bone volume could be due to either increase in bone formation or reduction in bone resorption, or both. On the basis of the observations described in the following paragraphs, the likely possibilities could include defects in osteoclastic activity to remodel the primary trabeculae.

\section{Osteoclastic bone resorption is downregulated in $k l / k l$ mutant mice}

Abnormal elongation of the trabeculae could be regarded as a result of reduced bone resorption at the trabecular bones. The numbers of osteoclasts (judged based on multinucleation with more than two nuclei and attachment to the bone surface in the lacunae) per tissue volume were about $50 \%$ less in $\mathrm{kl} / \mathrm{kl}$ mutants than the wild-type mice, whereas the bone surface of $k l / k l$ mutants, in which the trabecular bones are elongated, was three times more than that of the wild-type. Therefore, the number of osteoclasts per trabecular bone surface in the metaphyses is about one-sixth that in $\mathrm{kl} / \mathrm{kl}$ homozygotes compared with wild-type animals $(0.028$ compared with 0.18 respectively). Histological sections of the long bones stained for tartrate-resistant acid phosphatase (TRAP) showed that the number of TRAP positive cells was also less in $\mathrm{kl} / \mathrm{kl}$ mice than in controls.

It is possible that the defects in bone resorption are due to an anomaly in osteoclast formation. Cocultures of the primary osteoblastic cells prepared from calvariae with spleen or bone marrow cells indicated similar efficiency in TRAP-positive multinucleated cell (MNC) formation between the wild-type and $\mathrm{kl} / \mathrm{kl}$ homozygote mice $(36 \cdot 3$ and 36.5 cells per well respectively; Fig. 4). Although cocultures using bone marrow cells tended to yield slightly greater numbers of TRAP-positive MNCs in $\mathrm{kl} / \mathrm{kl}$ mice than in controls $(78.5$ compared with 55.8 cells per well), the difference was not large $(<20 \%)$ and not statistically significant. These results indicate that osteoblasts or osteoclast progenitors in mice lacking Klotho protein are not severely affected in their ability for osteoclastogenesis, at least in the ex vivo environment of the coculture experiments.

We also tested whether the TRAP-positive MNCs that were formed in our cocultures of $\mathrm{kl} / \mathrm{kl}$ mutant mouse-derived cells (osteoblasts and bone marrow) or wild-type mouse-derived cells (osteoblasts and bone marrow) could exhibit resorption activity. The TRAPpositive MNCs that developed in the cocultures exhibited pit formation activity. The in vitro resorption activities were similar between the TRAP-positive MNCs derived from the cocultures of $\mathrm{kl} / \mathrm{kl}$ mutant mouse cells and those derived from cocultures of wild-type mouse cells.

\section{Possible mechanisms for the abnormality in $k l / k l$ mutant mice}

As discussed above, the ratios of deep-blue-stained trabeculae to total trabecular bone volume were similar between wild-type and $\mathrm{kl} / \mathrm{kl}$ mutant mice, indicating that conversion from proteoglycan-rich trabeculae to bony trabeculae is taking place normally in the $k l / k l$ mutant mice. This finding suggests the following possible mechanisms for the abnormality in $\mathrm{kl} / \mathrm{kl}$ homozygotes. First, bone resorption by the osteoclasts in $\mathrm{kl} / \mathrm{kl}$ mutant mice might be impaired. In fact, the number of osteoclast-like MNCs in the tibiae of $\mathrm{kl} / \mathrm{kl}$ homozygote mice is less than that of the wild type. However, formation of osteoclast-like multinucleated TRAP-positive cells in coculture in vitro was observed to be similar in $\mathrm{kl} / \mathrm{kl}$ mutant- and wild-typederived cells. These in vitro data are in contrast to the data in vivo. One of the possible hypotheses is that the pathway in osteoclast formation may be affected in vivo in the $\mathrm{kl} / \mathrm{kl}$ mutant mice by the possible absence, or the presence, of certain humoral factor(s). This hypothesis is in accordance with the notion that klotho gene products could be released into the circulation (Matsumura et al. 1998, Shiraki-Iida et al. 1998) and that in situ hybridization did not show any klotho mRNA signals in bone. Our preliminary examination also failed to detect any klotho signal in bone, using Northern blot analysis, and Kuro-o et al. (1997) likewise failed to detect any klotho signal, even when they used RT-PCR. Another possible mechanism underlying the abnormality in the mutant mice is a relative acceleration of apoptosis in the osteoclasts in vivo, again by some humoral factor, the concentrations of which are affected by the mutation.

If osteoblastic bone formation activity is abnormally increased, then it could also cause elongation of the trabeculae seen in the $\mathrm{kl} / \mathrm{kl}$ mutant mice. However, this notion is not supported by our in vitro experiments, in that we examined the activity of osteoblastic nodule formation and subsequent calcium deposition by the cells in a long-term ( 3 weeks) culture in the presence of ascorbate and $\beta$-glycerophosphate. Nodule formation activity was similar between osteoblastic cells prepared from $\mathrm{kl} / \mathrm{kl}$ mutant calvaria and those from wild-type mice. Osteoblast-derived signal(s) would be required for osteoclastic maturation and activation to maintain coupling between the two types of cells in vivo. Although pit assay experiments using cocultured cells did not reveal differences between $\mathrm{kl} / \mathrm{kl}$ mutant cells and wild-type cells in vitro (Fig. 5), we cannot rule out the possibility that $\mathrm{kl} / \mathrm{kl}$ mutant mice have defects in the coupling mechanism in vivo.

Biochemical markers of bone metabolism in blood or urine are important, as the bones of the $\mathrm{kl} / \mathrm{kl}$ mutant mice revealed obvious abnormalities as described above. If any alterations could be found in serum concentrations of calcium, osteocalcin and bone-type alkaline phosphatase, 
and in urine pyridinoline and deoxypyridinoline, these data should give a clue to clarify the mechanism of the pathogenesis operating in $\mathrm{kl} / \mathrm{kl}$ mutant mice. These studies on the biochemical markers are currently under way.

Several genes involved in the pathogenesis of precocious aging in humans, such as in Werner's syndrome, have been identified to be modifiers of DNA structures. It would be interesting to see whether the klotho gene locus could be susceptible to these modifiers. Since Mgp knockout mice and $\mathrm{kl} / \mathrm{kl}$ mice share certain features, for example ectopic calcification in soft tissues such as aorta, we examined the expression of Mgp in the mice. Preliminary examination, however, did not show significant difference between $\mathrm{kl} / \mathrm{kl}$ mutant and wild-type mice in Mgp mRNA levels in these mice tissues.

\section{klotho mutant mice provide a novel type of skeletal abnormality model}

As mentioned before, $k l / k l$ mutant mice exhibit many features resembling age-associated diseases such as atherosclerosis and ectopic calcification in soft tissues. With regard to bones, osteoporosis in the diaphyses and the possible lower levels of resorption activity may be reflecting 'low-turnover' type human osteoporosis. However, establishment of direct relationship between the features seen in this $\mathrm{kl} / \mathrm{kl}$ mutant mice and osteoporosis in humans will be dependent upon further investigations.

Similarly, relationship between the high incidence of soft-tissue calcification in the $\mathrm{kl} / \mathrm{kl}$ mice and the corresponding age-associated calcification in humans (aorta, costal cartilage, etc.) should be further investigated. The abnormal elongation observed in the $k l / k l$ mice may be due to the nature of rodents, in that long bones in these animals are continuously growing, without clear closure of their growth plates as seen in humans. As long bones continuously maintain the growth plates and, although at slower rates, continue to provide trabecular bone extending into the metaphyseal regions in rodents, reduction of the bone-resorbing activity could result in the elongation of the trabecular bones. Therefore, the elongation part of the trabecular bones in mice may not have directly corresponding features in human age-associated bone changes. However, it is possible that, if a similar mechanism involving klotho gene mutation causes precocious aging in young humans who still have growth plates, elongation of the trabecular bones in the epiphyses would be observed. Nonetheless, it is at least clear that the mutation in this aging-related klotho gene has the effect of altering normal bone turnover.

\section{Conclusion}

In summary, these $\mathrm{kl} / \mathrm{kl}$ mice exhibit an elongated trabecular bone pattern in the epiphyseal regions, in association with relative osteopenia in the diaphysis. These dramatic changes in the trabecular bones of mice lacking expression of the klotho gene may predict a role for this newly identified gene product, Klotho, in novel aspects of the control of bone metabolism.

\section{Acknowledgements}

We thank Dr M Kuro-o for providing us with klotho transgenic mice and Drs K Tsuji, H N Matsumoto, and Ms N Kawaguchi for advice. This research was supported by a grant-in-aid received from the Ministry of Education (10044246, 10877233, 0930734, 07559096) and grants from CREST (Core Research for Evolutional Science and Technology) of Japan Science and Technology Corporation (JST), and a grant from the 'Research for the Future' Program of the Japan Society for the Promotion of Science (JSPS) (96I00205).

\section{References}

Ames BN, Shigenaga MK \& Hagen TM 1993 Oxidants, antioxidants, and the degenerative diseases of aging. Proceedings of the National Academy of Sciences of the USA 90 7915-7922.

Barlow C, Hirotsune S, Paylor R, Liyanage M, Eckhaus M, Collins F, Shiloh Y, Crawley JN, Ried T, Tagle D \& Wynshaw-Boris A 1996 Atm-deficient mice: a paradigm of ataxia telangiectasia. Cell 86 159-171.

Guarente L 1996 Do changes in chromosomes cause aging? Cell 86 9-12.

Henning KA, Li L, Iyer N, McDaniel LD, Reagan MS, Legerski R, Schultz RA, Stefanini M, Lehmann AR, Mayne LV \& Friedberg EC 1995 The Cockayne syndrome group A gene encodes a WD repeat protein that interacts with $\mathrm{CSB}$ protein and a subunit of RNA polymerase II TFIIH. Cell 82 555-564.

Kuro-o M, Matsumura Y, Aizawa H, Kawaguchi H, Suga T, Utsugi T, Ohyama Y, Kurabayashi M, Kaname T, Kume E, Iwasaki H, Iida A, Shirakiiida T, Nishikawa S, Nagai R \& Nabeshima Y 1997 Mutation of the mouse klotho gene leads to a syndrome resembling ageing. Nature 390 45-51.

Luo G, Ducy P, McKee MD, Pinero GJ, Loyer E, Behringer RR \& Karsenty G 1997 Spontaneous calcification of arteries and cartilage in mice lacking matrix GLA protein. Nature $38578-81$.

Matsumura Y, Aizawa H, Shirakiiida T, Nagai R, Kuroo M \& Nabeshima Y 1998 Identification of the human klotho gene and its two transcripts encoding membrane and secreted klotho protein. Biochemical and Biophysical Research Communications 242 626-630.

Salazar M, Leong T, Tu N, Gelman RS, Watson AL, Bronson R, Iglesias A, Mann M, Good RA \& Yunis EJ 1995 Life-span, T-cell responses, and incidence of lymphomas in congenic mice. Proceedings of the National Academy of Sciences of the USA 92 3992-3996.

Savitsky K, Bar-Shira A, Gilad S, Rotman G, Ziv Y, Vanagaite L, Tagle DA, Smith S, Uziel T, Sfez S, Ashkenazi M, Pecker I, Frydman M, Harnik R, Patanjali SR, Simmons A, Clines GA, Sartiel A, Gatti RA, Chessa L, Sanal O, Lavin MF, Jaspers NGJ, Taylor AMR, Arlett CF, Miki T, Weissman SM, Lovett M, Collins FS \& Shiloh Y 1995 A single ataxia telangiectasia gene with a product similar to PI-3 kinase. Science 268 1749-1753.

Shiraki-Iida T, Aizawa H, Matsumura Y, Sekine S, Iida A, Anazawa H, Nagai R, Kuroo M \& Nabeshima Y 1998 Structure of the mouse klotho gene and its two transcripts encoding membrane and secreted protein. FEBS Letters 424 6-10. 
Troelstra C, van Gool A, de Wit J, Vermeulen W, Bootsma D \& Hoeijmakers JH 1992 ERCC6, a member of a subfamily of putative helicases, is involved in Cockayne's syndrome and preferential repair of active genes. Cell 71 939-953.

Yamashita T, Kuro-o M, Nabeshima Y \& Noda M 1998 Severe hypercalcification associated with precocious aging in transgenic mouse carrying insertional mutation in a novel Klotho gene locus. Experimental Medicine 16 131-135
Yu CE, Oshima J, Fu YH, Wijsman EM, Hisama F, Alisch R, Matthews S, Nakura J, Miki T, Ouais S, Martin GM, Mulligan J \& Schellenberg GD 1996 Positional cloning of the Werner's syndrome gene. Science 272 258-262.

Received 8 April 1998

Accepted 15 May 1998 